\title{
A puzzling paucity of double peaked X-ray pulsars
}

\author{
T. Bulik ${ }^{1}$, D. Gondek-Rosińska ${ }^{2,1}$, A. Santangelo ${ }^{3}$, T. Mihara ${ }^{4}$, M. Finger ${ }^{5}$, and M. Cemeljic ${ }^{6}$ \\ 1 Nicolaus Copernicus Astronomical Center, Bartycka 18, 00716 Warsaw, Poland \\ ${ }^{2}$ LUTH, Observatoire de Paris, Place Jules Janssen, 92195 Meudon Cedex, France \\ 3 IFCAI-CNR, via Ugo La Malfa 153, 90146 Palermo, Italy \\ ${ }^{4}$ RIKEN, 2-1 Hirosawa, Wako, Saitama 351-0198, Japan \\ 5 NSSTC, 320 Sparkman Dr., Hunstville, AL 35805, USA \\ 6 AIP, an der Sternwarte 16, 14482 Potsdam, Germany
}

Received 19 November 2002 / Accepted 25 March 2003

\begin{abstract}
Accretion powered pulsars exhibit a variety of lightcurves. In this paper we propose to classify the observed lightcurves as single or double pulsed. We analyze the lightcurves of 89 accretion powered pulsars and assign them to these classes. We present three datasets: first in which the classification can be easily done, second for which the classification is more difficult and not certain, and third for which we were unable to classify the pulsar because of a lack of published data. We analyze a simple model in which the angles between the magnetic and rotation axis $\beta$, and between the rotation axis and the line of sight $\theta$ are random, and show that it is inconsistent with the data. We also present a model in which the angle between the magnetic axis and the rotation axis is restricted and compare it with the data. This leads to an upper limit on the angle $\beta<50^{\circ}$. We conclude that there must be a mechanism that leads to alignment of the magnetic and spin axis in X-ray pulsars.
\end{abstract}

Key words. X-rays: star - stars: neutron

\section{The lightcurves of accretion powered X-ray pulsars}

Accreting neutron stars were discovered more than 30 years ago (Shklovsky 1967), with Cen X-3 beeing the first one discovered that showed pulsations (Giacconi et al. 1971). Currently we know nearly one hundred accreting neutron stars, and in more than eighty of them pulsations were identified (Liu et al. 2000, 2001). Accreting neutron stars in binaries exhibit a wide range of X-ray light curves. They vary as a function of the photon energy, and moreover in the transient sources the pulse shapes change with the variation of the luminosity.

The pulse period in accreting sources is identified with the rotation of a magnetized star. As the matter from the companion star falls onto the neutron star it is channeled onto the magnetic poles by the strong magnetic field of the neutron star. Thus the polar caps and/or accretion columns are the places where most of the emission takes place. Several theoretical models of radiation of magnetized accretion powered neutron stars have been proposed and the beam shape is usually described in terms of pencil beams, when most of the radiation is emitted along the magnetic field, or fan beams when most of the radiation is emitted perpendicularly to the magnetic field.

The magnetized radiative transfer is solved using a difference scheme (Meszaros \& Nagel 1985; Bulik et al. 1992), or using Monte Carlo scheme (Lamb et al. 1990;

Send offprint requests to: T. Bulik, e-mail: bulik@camk.edu.pl
Isenberg et al. 1998), for a review see Meszaros (1992). In the pencil beam model radiation from each polar cap produces one pulse in the light curve. Depending on the emission cap physics and the strength of the magnetic field each pulse may have some additional structure. When during the rotation of an accreting pulsar we see two polar caps the lightcurve should exhibit two distinct pulses (peaks), and if only one cap is seen then the lightcurve is single peaked. In the case of dominant radiation from the accretion column (fan beam model) the shape of the lightcurve will depend on the geometry and on the shape of the beam. Even with simple beam shapes there can be triple or quadruple peaked lightcurves.

Each pulsar can be classified according to the number of peaks in the lightcurve depending on the location of the observer and the inclination between the rotation and the magnetic axes. We note that the observed pulsar lightcurves can be classified into two groups: the single pulsed light curves and the double pulsed light curves. At low X-ray energies this distinction may not be clear, however in the high energy band we clearly distinguish single or double pulses, and there are no triple (or multiple) pulsed lightcurves, see e.g. Nagase (1989); Bildsten et al. (1997). By high energy band we mean at least above $10 \mathrm{keV}$, or in the pulsars where cyclotron lines were found, above the cyclotron lines.

The paper is organized as follows: in Sect. 2 we summarize the observations, in Sect. 3 we analyze the expected shapes and expected fraction of single and double peaked lightcurves, in 
Sect. 4 we discuss the results. We summarize the conclusions in Sect. 5.

\section{Pulsar observations}

In this section we review the existing observations of X-ray pulsars and attempt to classify their lightcurves according to the number of pulses in their lightcurves. We summarize our classification in Tables 1, 2, and 3. For each pulsar we have searched the literature for X-ray observations, and verified if the pulsar was observed in the band above $10 \mathrm{keV}$, or above the cyclotron line energy. The pulsars with such observations are listed in Table 1 . There are 46 entries in the Table out of which 20 have been classified as double and 26 as single. Additionally we have listed 31 pulsars in Table 2 for which the classification was difficult. For some of the pulsars no lightcurve above $10 \mathrm{keV}$ was available, we have also included here three pulsars with accretion flow eclipses (Cemeljic \& Bulik 1998) that is pulsar with the light curves exhibiting sudden dips. Such dips can be interpreted as accretion flow eclipses, i.e. be caused by the absorption of the X-ray flux when the accretion column passes the line of sight. Such phenomena have been noted for at least three objects: A0535-262 (Cemeljic \& Bulik 1998), GX1+4 (Giles et al. 2000), and RX J0812.4-3114 (LS992) (Reig \& Roche 1999b). We classified them conservatively as double, although we rather lean toward the interpretation of single peaks with an accretion flow eclipse. There are 7 pulsars with likely double peaked light curves and 24 with single in Table 2 . We see that in each table there are more single pulsed than double pulsed objects. For completion we also list 13 pulsars in Table 3 for which no information on the type of the lightcurve was available. We define three sets of data corresponding to the three tables: dataset $\mathcal{S}_{1}$ with the data of Table 1 , dataset $\mathcal{S}_{2}$ with the combined data of Tables 1 and 2 , and dataset $\mathcal{S}_{3}$ with all the data, and the entries of Table 3 are assumed to be double peaked.

\section{The expected pulsars light curves}

Let us assume that an accreting neutron star has a pure centered dipole field. In such a case the geometry of accretion powered pulsars is characterized by two angles: $\theta$ - the angle between the rotation axis and direction to the observer, and $\beta$ the angle between the magnetic and rotation axes. Within the pencil beam model the parameter space when only one cap is seen is determined by the condition:

$\theta<90-\beta-\Delta_{\mathrm{g}}$,

where $\Delta_{\mathrm{g}}$ is the gravitational light deflection of a ray emitted parallel to the surface of the neutron star. Here we assume that the magnetic beaming is not very strong and that there are $\mathrm{X}$ ray photons emitted parallel to the surface of the neutron star. Ignoring the gravitational light bending effects, i.e. in the classical Newtonian case, $\Delta_{\mathrm{g}}=0^{\circ}$, the parameter space for seeing just one cap is shown as the region above the solid line in Fig. 1. Thus, if the angles $\beta$ and $\theta$ are chosen randomly the fraction of single pulsed objects should be $1-\pi / 4 \approx 0.214$. The region

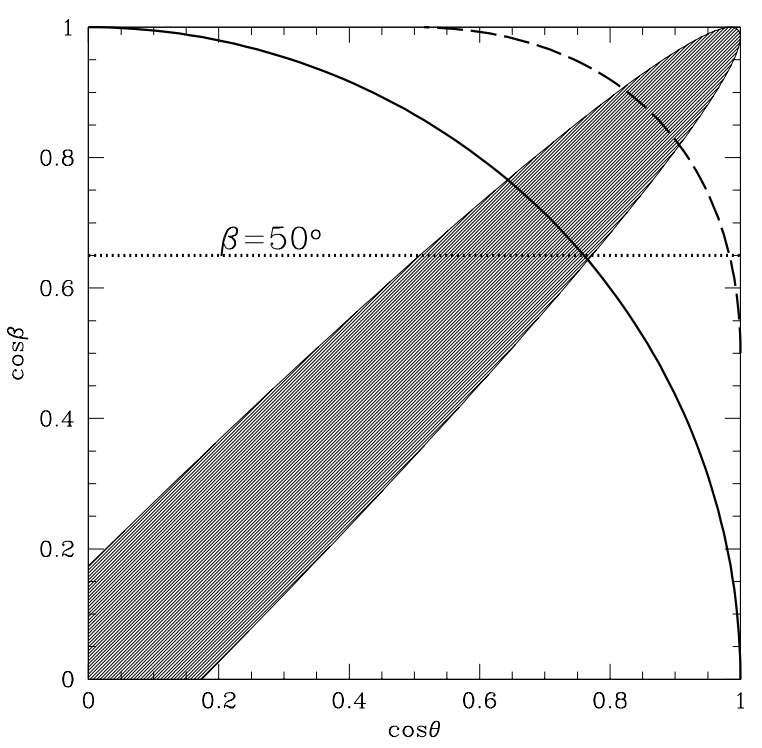

Fig. 1. Parameter space for viewing accreting X-ray pulsars. The region below the solid line corresponds to visibility of two polar caps with gravitational light bending neglected. The region below the dashed line corresponds to visibility of two caps with the gravitational light deflection $\Delta_{\mathrm{g}}=30^{\circ}$. The shaded region is the part of the parameter space in which pulses would be detectable if radiation is strongly beamed. The horizontal dotted line corresponds to the angle between the rotation and magnetic axes $\beta=50^{\circ}$.

for the single peaked light curves would be smaller if the radiation were strongly beamed. However, in this case the double peaked light curves would exhibit narrow pulses, contrary to what is observed - see e.g. Fig. 7 in Bildsten et al. (1997).

The angles $\beta$ and $\theta$ also determine the number of pulses in the lightcurve within the fan beam model. However here the number of pulses seen depends also on the shape of the beam. In a simple case when there are two columns radiating with constant specific intensity the beam will be a function only of the angle $\psi$ between the magnetic axis and the direction to the observer: $F_{1}(\psi) \propto|\sin \psi|$. Interestingly, the parameter space for seeing two peaked lightcurves is identical to the case of the pencil beam model discussed above, the only difference being that for the given angles $\beta$ and $\theta$ the maxima of the lightcurve fall at different phases. Introducing a more general beam pattern e.g. $F_{2}(\psi) \propto|\sin 2 \psi|$ leads to dominance of multiple peaked lightcurves: in this case we expect $4.5 \%$ single peaked, $27.8 \%$ double peaked, $40.1 \%$ triple peaked, and $27.6 \%$ quadruple peaked lightcurves.

For a set of data, like pulsars, with a well defined property, i.e. either single or double pulsed, and a given probability $p$ that each pulsar will belong to one or other class, the probability of observing $n$ single peaked lightcurves and $m$ double peaked in an observation of randomly chosen $n+m$ objects is given by the binomial distribution:

$P_{n, m}=\frac{(n+m) !}{n ! m !} p^{n}(1-p)^{m}$ 
Table 1. X-ray pulsars in group one, i.e. these that are easily classifiable.

\begin{tabular}{|c|c|c|c|c|}
\hline System & $P_{\text {spin }}$ & Type & Comments & References \\
\hline SAX J1808.4-3658 & 0.002494 & Single & & Wijnands \& van der Klis (1998) \\
\hline EXO 1024.0-5732 & 0.061 & Double & & Caraveo et al. (1989) \\
\hline GRO J1744-28 & 0.467 & Single & The Bursting Pulsar & Finger et al. (1996) \\
\hline 2A $1822-371$ & 0.592 & Double & & Jonker \& van der Klis (2001) \\
\hline SMC X-1 & 0.717 & Double & & Levine et al. (1993) \\
\hline Her X-1 & 1.24 & Double & 2nd pulse weak & Tananbaum et al. (1972); Bildsten et al. (1997) \\
\hline SMC X-2 & 2.37 & Single & & Corbet et al. (2001) \\
\hline $4 \mathrm{U} 0115+63$ & 3.61 & Single & & Santangelo et al. (1999) \\
\hline V0332+53 & 4.37 & Single & & Makishima et al. (1990) \\
\hline GRO J1750-27 & 4.45 & Single & & Scott et al. (1997) \\
\hline Cen $\mathrm{X}-3$ & 4.82 & Double & 2nd pulse weak & Giacconi et al. (1971); Burderi et al. (2000) \\
\hline $1 \mathrm{E} 2259+586$ & 6.98 & Double & & Iwasawa et al. (1992) \\
\hline 4U 1626-67 & 7.66 & Single & & Giacconi et al. (1972); Chakrabarty et al. (1997) \\
\hline GS 0834-430 & 12.3 & Double & & Aoki et al. (1992); Wilson et al. (1997) \\
\hline LMC X-4 & 13.4 & Single & & Tananbaum et al. (1972); Woo et al. (1996) \\
\hline J00521-7319 & 15.3 & Double & & Finger et al. (2001) \\
\hline XTE J1946+274 & 15.8 & Double & & Paul et al. (2001) \\
\hline 2S $1417-624$ & 17.6 & Double & & Bildsten et al. (1997) \\
\hline GRO J1948+32 & 18.7 & Single & & Chakrabarty et al. (1995) \\
\hline RX J0117.6-7330 & 22.0 & Double & & Macomb et al. (1999) \\
\hline GS $1843+00$ & 29.5 & Double & & Koyama et al. (1990b) \\
\hline J0112.2-7317 & 31.03 & Single & & Wilson \& Finger (1998) \\
\hline OAO 1657-415 & 37.7 & Single & & Bildsten et al. (1997) \\
\hline EXO 2030+375 & 41.7 & Double & broad peak with two maxima & Stollberg et al. (1999); Reig \& Coe (1999) \\
\hline 1SAX J0054.9-7226 & 59 & Single & & Santangelo et al. (1998) \\
\hline Cep X-4 & 66.2 & Double & & Koyama et al. (1991a) \\
\hline XTE J1906+09 & 89.2 & Single & double peak below $20 \mathrm{keV}$ & Marsden et al. (1998) \\
\hline GRO J1008-57 & 93.5 & Single & & Bildsten et al. (1997) \\
\hline GS $1843-024$ & 94.8 & Single & & Koyama et al. (1990c) \\
\hline 4U 0728-25 & 103.2 & Double & & Corbet \& Peele (1997) \\
\hline Sct X-1 & 111 & Double & broad peak two maxima & Koyama et al. (1991b) \\
\hline GRO J2058+42 & 198 & Single & & Bildsten et al. (1997) \\
\hline XTE J1858+034 & 202.7 & Single & & Paul \& Rao (1998) \\
\hline Vela X-1 & 283 & Double & & Bulik et al. (1995); Bildsten et al. (1997) \\
\hline 4U 1145-619 & 292 & Single & & Saraswat et al. (1997); Bildsten et al. (1997) \\
\hline 1E $1145.1-6141$ & 297 & Single & & Grevenev et al. (1992) \\
\hline J0103.2-7209 & 345.2 & Single & & Israel et al. (2000) \\
\hline SAX J2103.5+4545 & 358.6 & Single & & Hulleman et al. (1998) \\
\hline XTE J1855-026 & 361.1 & Single & & Corbet et al. (1999b) \\
\hline A $1118-616$ & 405 & Single & & Coe et al. (1994) \\
\hline GPS $1722-363$ & 414 & Single & & Tawara et al. (1989) \\
\hline 4U 1907+09 & 438 & Double & 2nd pulse weak & in 't Zand et al. (1998) \\
\hline 4U $1538-52$ & 530 & Double & & Bildsten et al. (1997) \\
\hline GX 301-2 & 681 & Double & & Koh et al. (1997) \\
\hline X Per & 835 & Single & & Robba \& Warwick (1989); Robba et al. (1996) \\
\hline RX J0146.9+6121 & 1413 & Single & & Mereghetti et al. (2000) \\
\hline
\end{tabular}

and the probability of obtaining $M$ or fewer double peaked objects in a sample containing $N$ sources is a sum

$\mathcal{P}_{N, M}=\sum_{m=1}^{M} P_{N-m, m}$.
We can test the consistency of the datasets defined above with the simplest model: random inclination of the magnetic axis to the rotation axis $\beta$, random direction of the line of sight $\theta$, and no gravitational light deflection. For the dataset $\mathcal{S}_{1}$ we obtain 
Table 2. X-ray pulsars in group two, i.e. with no pulse shape above $10 \mathrm{keV}$ or were difficult to classify.

\begin{tabular}{|c|c|c|c|c|}
\hline System & $P_{\text {spin }}$ & Type & Comments & References \\
\hline A0538-67 & 0.069 & Double & Einstein obs & Skinner et al. (1982) \\
\hline SAX J0635+0533 & 0.0338 & Single & below $10 \mathrm{keV}$ & Cusumano et al. (2000) \\
\hline RX J0059.2-7138 & 2.76 & Single & ROSAT obs & Hughes (1994) \\
\hline RX J0502.9-6626 & 4.06 & Single & ROSAT obs & Schmidtke et al. (1995) \\
\hline RX J1838.4-0301 & 5.45 & Single & ROSAT obs & Schwentker (1994) \\
\hline 1E $1048-593$ & 6.44 & Single & Einstein obs & Seward et al. (1986) \\
\hline AX J1845.0-0300 & 7 & Single & ASCA obs & Torii et al. (1998b) \\
\hline RX J0720.4-3125 & 8.39 & Single & XMM obs & Cropper et al. (2001) \\
\hline $4 \mathrm{U} 0142+614$ & 8.69 & Double & obs below $10 \mathrm{keV}$ & Israel et al. (1994) \\
\hline 2E 0050.1-7247 & 8.88 & Single & ROSAT obs & Israel et al. (1995) \\
\hline 2S $1553-54$ & 9.26 & Double & SAS-3 obs below $11 \mathrm{keV}$ & Kelley et al. (1983) \\
\hline AX J0049-732 & 9.31 & Single & ASCA obs & Ueno et al. (2000) \\
\hline 1RXS 170849.0-400910 & 11.0 & Single & ASCA obs; could be an AnXP & Sugizaki et al. (1997) \\
\hline RX J0648.1-4419 & 13.2 & Single & ROSAT obs & Israel et al. (1997) \\
\hline XTE J0111.2-7317 & 30.95 & Single & ASCA obs & Yokogawa et al. (2000b) \\
\hline RX J0812.4-3114 & 31.9 & Double & accretion flow eclipse & Reig \& Roche (1999b) \\
\hline RX J0529.8-6556 & 69.5 & Single & ROSAT obs & Haberl et al. (1997) \\
\hline AX J0049-729 & 74.8 & Single & ASCA obs & Yokogawa et al. (1999) \\
\hline SAX J0544.1-710 & 96.1 & Single & obs below $10 \mathrm{keV}$ & Cusumano et al. (1998) \\
\hline $\mathrm{A} 0535+26$ & 105 & Double & accretion flow eclipse & Bildsten et al. (1997) \\
\hline GX $1+4$ & 120 & Double & accretion flow eclipse & Bildsten et al. (1997) \\
\hline 1SAX J1324.4-6200 & 170 & Single & SAX obs below $20 \mathrm{keV}$ & Angelini et al. (1998) \\
\hline RX J0440.9+4431 & 202.5 & Single & obs below $20 \mathrm{keV}$ & Reig \& Roche (1999a) \\
\hline AX J1749.2-2725 & 220 & Single & ASCA obs & Torii et al. (1998a) \\
\hline GX 304-1 & 272 & Single & SAS-3 obs & McClintock et al. (1977) \\
\hline AX J0058-7203 & 280 & Double & ASCA obs & Tsujimoto et al. (1999) \\
\hline AX J0051-733 & 323 & Single & ASCA obs & Imanishi et al. (1999) \\
\hline 1SAX J1452.8-5949 & 437 & Single & obs MECS below $10 \mathrm{keV}$ & Oosterbroek et al. (1999) \\
\hline AX J170006-4157 & 714.5 & Single & ASCA obs & Torii et al. (1999) \\
\hline RX J1037.5-564 & 860 & Single & pulse shape below $20 \mathrm{keV}$ & Reig \& Roche (1999a) \\
\hline $2 \mathrm{~S} 0114+650$ & 10008 & Single & ASM obs below $12 \mathrm{keV}$ & Corbet et al. (1999a); Hall et al. (2000) \\
\hline
\end{tabular}

Table 3. X-ray pulsar in group three, for which no reliable pulse shapes were found.

\begin{tabular}{lllll}
\hline \hline System & $P_{\text {spin }}$ & Type & Comments & References \\
\hline J0501.1-7211 & 3.343 & $?$ & no lc published & Yokogawa \& Koyama (1998) \\
EXO 053109-6609.2 & 13.7 & $?$ & EXOSAT detection & Dennerl et al. (1995) \\
J0053.8-7226 & 46.6 & $?$ & no lc published & Corbet et al. (1998) \\
X 1839-04 & 81.1 & $?$ & no lc published & Koyama et al. (1990a) \\
J0051-722 & 91.1 & $?$ & & Liu et al. (2001) \\
AX 1820.5-1434 & 152 & $?$ & weak pulsations & Kinugasa et al. (1998) \\
J0054-720 & 169.3 & $?$ & no lc published & Lochner et al. (1998) \\
3U 1239-599 & 191 & $?$ & Ariel obs, no lc published & Huckle et al. (1977) \\
XTE J1858+034 & 221 & $?$ & no lc published & Remillard et al. (1998) \\
4U 2206 + 54 & 392 & $?$ & no lc published & Saraswat \& Apparao (1992) \\
1WGA J1958.2+3232 & 730 & $?$ & only ROSAT lc & Israel et al. (1998) \\
AX J0049.5-7323 & 755 & $?$ & weak pulsations & Yokogawa et al. (2000a) \\
\hline
\end{tabular}

the probability $2.2 \times 10^{-7}$ that there are 20 or fewer double pulsars in a set of 46 observed objects. Applying this test to the dataset $\mathcal{S}_{2}$ we obtained the probability of only $2.5 \times 10^{-16}$, and for the dataset $\mathcal{S}_{3}$ this probability is $9.2 \times 10^{-13}$. Thus we see that the data are inconsistent with this simple model. This is due to the fact that the parameter space for viewing single pulsed objects is smaller than for the double pulsed objects. This result holds for both the pencil beam and fan beam $F_{1}$. For the fan beam of the form $F_{2}$ the results are even more significant since probability of seeing single pulsed pulsars is even smaller: for 


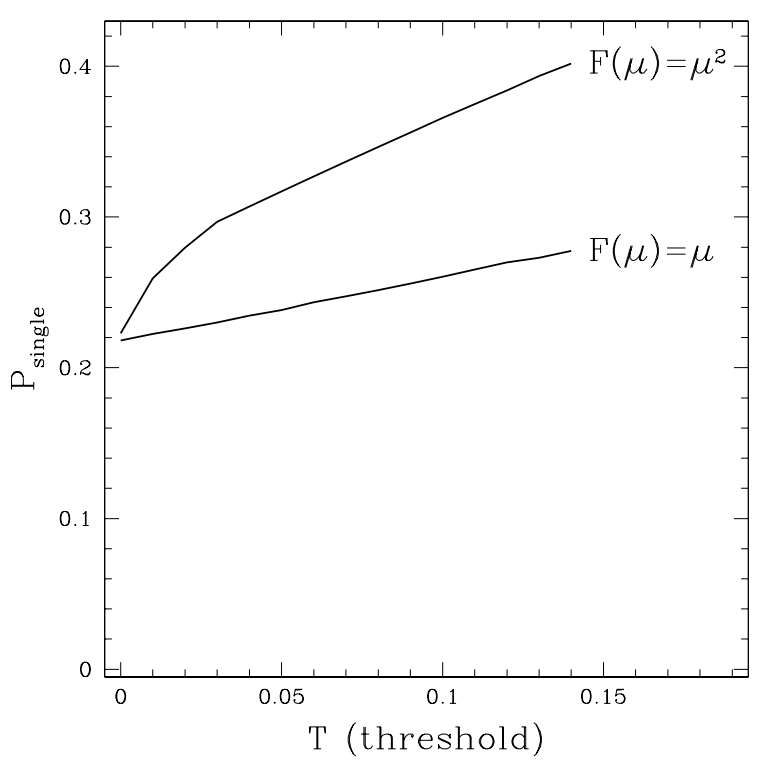

Fig. 2. The probability of classifying a pulsar as single peaked as a function of the threshold below which the second peak is not detectable.

the set $\mathcal{S}_{1}$ we obtain the probability $1.8 \times 10^{-22}$, for $\mathcal{S}_{2}$ the probability is $3.7 \times 10^{-46}$, and for $\mathcal{S}_{3}$ it is $1.2 \times 10^{-41}$. In this analysis we assumed that all multiple peaked model lightcurves fall into the two peaked class. We note, however, that the fan beam model predicts triple and quadruple peaked lightcurves which are not observed.

The real detectability of the second peak depends on the exact shape of the beam and on the strength of the signal. The second peak may be a small bump in the pulse shape and when the signal to noise is low it might be undetected. General modeling of second peak detectability is difficult because it must include the unknown beam shape and the distribution of signal to noise ratios. Here we present a simple model in which we assume that we can detect the second peak if its amplitude is at least a certain fraction of the main peak. Furthermore we assume that the beam flux is described either by $F(\mu)=\mu$, or $F(\mu)=\mu^{2}$, where $\mu=\cos \psi$ is the cosine of the angle between the normal and the line of sight, and that the pulsar is detectable from every direction. For these two beam shapes we calculate the pulse shapes as a function of the angles $\beta$ and $\theta$. For each pulse shape we calculate $R_{2}$ the ratio of the magnitude of the second peak in relation to the main one. We then define a detection threshold $T$ for the second peak: if $R_{2}>T$ a pulse shape is classified as a double and otherwise as a single. We calculate the probability of classifying a pulsar as single pulsed as a function of the detection threshold $T$ for the two assumed beam shapes and plot the results in Fig. 2. The probability of classifying a pulsar as single peaked increases with increasing the threshold $T$, the case $T=0$ corresponds to the ideal case when even the smallest second peaks are detectable. When $T=0.1$ the value of $P_{\text {single }}$ reaches $P_{\text {single }}=0.26$ for the $F(\mu)=\mu$ beam and $P_{\text {single }}=0.36$ for the $F(\mu)=\mu^{2}$ beam. We would have classified the pulse shapes as double peaked with the second peak as small as a few percent of the main peak amplitude. We take the case $T=0.1$, together with the $F(\mu)=\mu^{2}$ beam i.e.
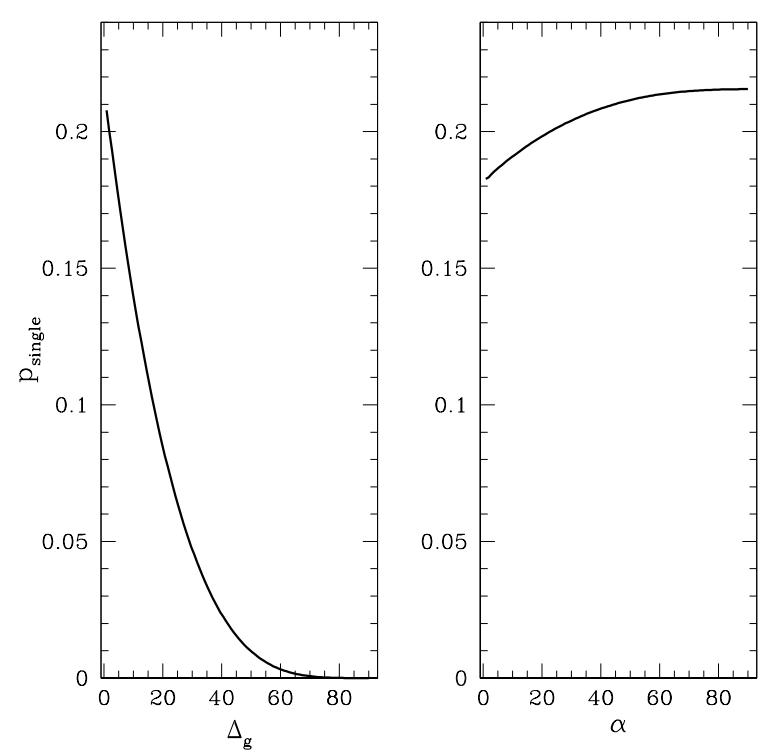

Fig.3. Left panel: the probability of seeing only one polar cap as a function of the maximal gravitational light deflection. The nonrelativistic case corresponds to $\Delta_{\mathrm{g}}=0$. Right panel; the probability of seeing only one cap as a function of the beaming angle $\alpha$.

$P_{\text {single }}=0.36$ and find that the probabilities of obtaining the observed datasets are $3.6 \times 10^{-3}$ for the $\mathcal{S}_{1}, 2.4 \times 10^{-7}$ for $\mathcal{S}_{2}$, and $8.4 \times 10^{-5}$ for $\mathcal{S}_{3}$. We note that we have assumed that a pulsar is detectable from all directions, while for strongly beamed cases there is a fraction of the parameter space where a pulsar is undetectable because of small flux. In the calculations below we consider the model with $T=0$.

Including the effects of the gravitational light deflection only reduces the probability of seeing one pulsed objects and makes the values of the above probabilities smaller. We present the probability of seeing one polar cap as a function of $\Delta_{\mathrm{g}}$ in the left panel of Fig. 3. Another possibility to consider is that the pulsar beam is not wide but strongly collimated, and the beam has an opening angle of $\alpha$. In this case for a given $\beta$ there is only a small range of angles $\beta-\alpha<\theta<\beta+\alpha$ for which the pulsar is visible at all. The restriction on the parameter space for small $\alpha=10^{\circ}$ is shown in Fig. 1 as the shaded region. The probability of seeing only one accretion cap as a function of the angle $\alpha$ is shown in the right panel of Fig. 3. Another possibility to consider is to allow for non aligned dipole, i.e. to assume that the two polar caps are not antipodal but can be placed anywhere on the stellar surface. A straightforward calculation neglecting the gravitational light bending effects shows that the probability of seeing just one polar cap drops in this case to $17 \%$. Thus the probabilities computed above are conservative and inclusion of the gravitational light bending and beaming make the result even more significant.

The effects of gravitational light deflection in the case of fan beams do not alter the results. We calculate the beam shape modified by the gravitational light bending using the approximate formulae of Beloborodov (2002). For the simple fan beam $F_{1}$ the probability of seeing the single or double peaked lightcurves does not depend on $\Delta_{\mathrm{g}}$. This is due to the fact that gravitational light bending does not change qualitatively the 


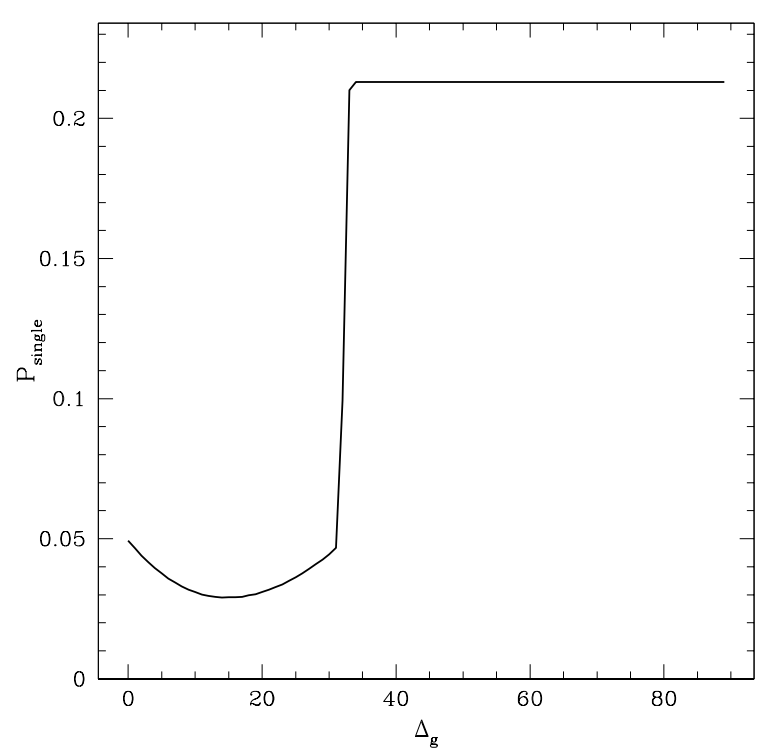

Fig. 4. The probability of seeing only one polar cap as a function of the maximal gravitational light deflection when the beam is described by the from $F_{2}$. The non-relativistic case corresponds to $\Delta_{\mathrm{g}}=0$.

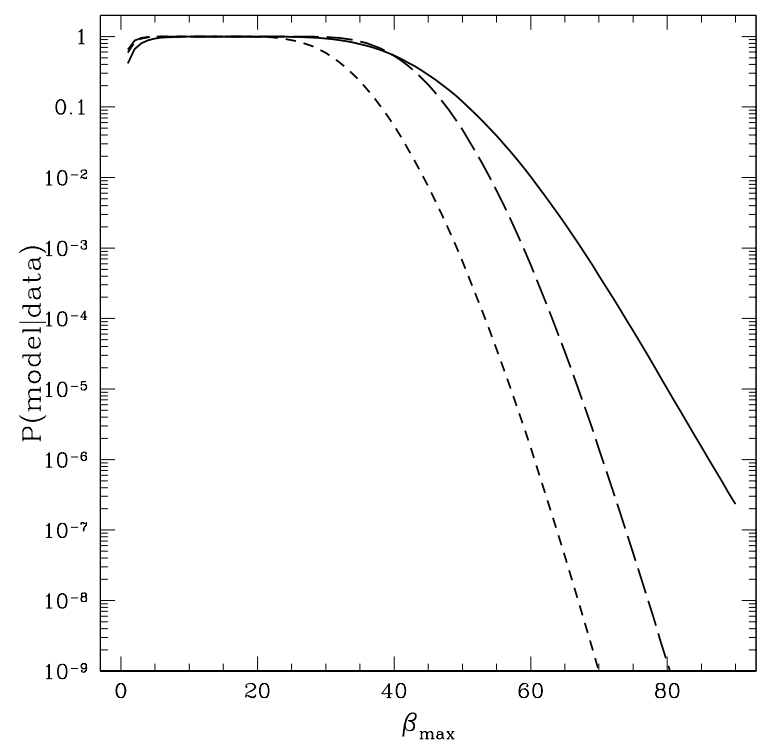

Fig. 5. The probability of observing the data in models pencil beam parameterized by the the maximum angle between the magnetic and rotation axis $\beta_{\max }$. The solid line corresponds to the data set $\mathcal{S}_{1}$, and the short-dashed line corresponds to the data set $\mathcal{S}_{2}$. The long-dashed line corresponds to the data set $\mathcal{S}_{3}$.

shape of the beam: it has a minimum along the magnetic axis and a single maximum in the direction perpendicular to the magnetic axis. We present the probability of seeing a single pulsed lightcurve for the fan beam of the form $F_{2}$ in Fig. 4. Here the shape of the beam depends strongly on the value of $\Delta_{\mathrm{g}}$. For small values of $\Delta_{\mathrm{g}}$ the probability of seeing single pulsed lightcurves slightly decreases, and then it rises to 0.21 at $\Delta_{\mathrm{g}} \approx 30^{\circ}$. At this point the gravitational light bending causes the two beams from the two columns to merge and the beam shape is qualitatively the same as for the case $F_{1}$.

The incompatibility of the simple model with the data prompts us to consider a more general model. We assume first

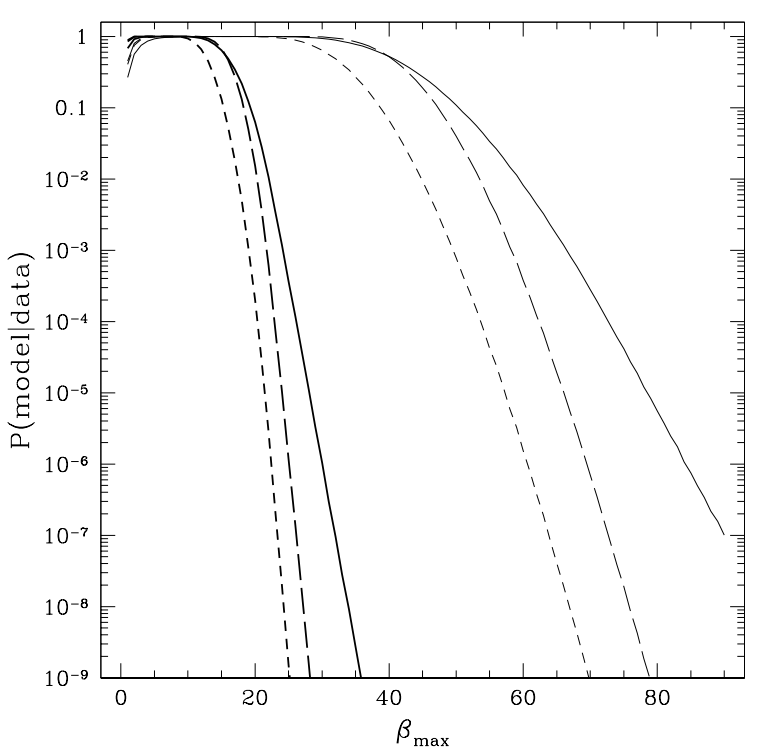

Fig. 6. The probability of observing the data in fan beam models parameterized by the the maximum angle between the magnetic and rotation axis $\beta_{\max }$ : thin lines - fan beam $F_{1}$, thick lines - fan beam $F_{2}$. The solid line corresponds to the data set $\mathcal{S}_{1}$, and the short-dashed line corresponds to the data set $\mathcal{S}_{2}$. The long-dashed line corresponds to the data set $\mathcal{S}_{3}$.

that the distribution of the inclinations of the magnetic axes to the rotation axis is restricted, i.e. the angles $\beta$ are limited to the range between 0 and $\beta_{\max }$. This corresponds to restricting the parameter space to the region above a horizontal line at $\cos \beta_{\max }$ in Fig. 1. For each value of $\beta_{\max }$ we can compute the probability of observing the given set of data using Eq. (3). We plot this probability as a function of $\beta_{\max }$ for the case of pencil beam in Fig. 5 and for the two fan beams considered in Fig. 6 . The standard model corresponds to the case $\beta_{\max }=90^{\circ}$. In Fig. 5 the probability of seeing the data rises to nearly unity for the angles $\beta_{\max }<40^{\circ}$ for the datasets $\mathcal{S}_{1}$ and $\mathcal{S}_{3}$, and $\beta_{\max }<30^{\circ}$ for the dataset $\mathcal{S}_{2}$ in the case of pencil beam model of Fig. 5. The probabilities of seeing one cap increases with decreasing $\beta_{\max }$, and therefore for its intermediate values the probability of seeing the data is rising to nearly unity. For the very small values of $\beta_{\max }$ one expects to see nearly all pulsars as single pulsed. Since each dataset contains some double pulsed objects the probability of seeing the data drops when $\beta_{\max } \rightarrow 0$. In the case of fan beam $F_{2}$ (Fig. 6) the probability of seeing the data rises to unity when $\beta_{\max }<15^{\circ}$. For this shape of the beam there is a significant part of the parameter space where the expected lightcurves are multiple peaked but we note that there are no multiple peaked lightcurves in the parameter space restricted to small angles $\beta$. The triple peaked lightcurves appear when $\beta>23^{\circ}$ and quadruple peaked ones when $\beta>46^{\circ}$. Thus in the framework of the fan beam model we do not expect multiple peaked pulse shapes if the inclination of the magnetic and rotation axes is limited to small angles only.

A more general model taking into account the general relativistic effects includes two parameters: the maximal angle between the magnetic and rotation axis $\beta_{\max }$ and the maximum gravitational light deflection angle $\Delta_{\mathrm{g}}$. In the framework of the pencil beam model we compute the probability of seeing 

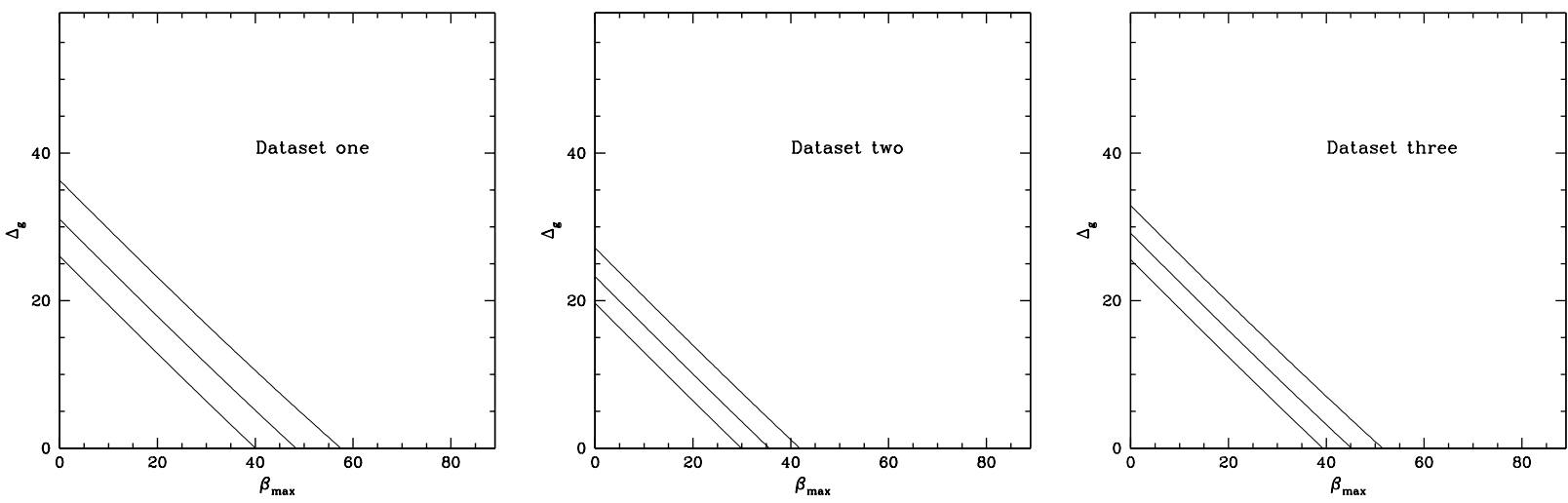

Fig. 7. Each panel presents the region containing the models that are consistent with the data at the level of $60 \%$, $90 \%$, and $99 \%$. The three panels correspond to the three datasets.

a pulsar with only one polar cap as a function of these two angles. In Fig. 7 we present the regions containing the models that are consistent with the data with the probability higher than $40 \%, 10 \%$, and $1 \%$. The non relativistic results correspond to the line $\Delta_{\mathrm{g}}=0$ in Fig. 7 . Since the probability of seeing only one polar cap decreases with increasing $\Delta_{\mathrm{g}}$, we also set a limit on its maximum value. The region corresponding to the $1 \%$ agreement limit for the dataset $\mathcal{S}_{1}$ can be expressed as $\Delta_{\mathrm{g}}+0.63 \beta_{\max }<36^{\circ}$. Thus if there were no gravitational light deflection effects we find that the inclination angle of the magnetic axis to the rotation axis should be smaller than $\approx 50^{\circ}$. For the case with a moderate gravitational light bending $\Delta_{\mathrm{g}}=30^{\circ}$ the magnetic axis inclination must be smaller than $\beta_{\max }<10^{\circ}$. We also see that the amount of gravitational light bending cannot exceed $36^{\circ}$ at the $99 \%$ confidence level. The limits are even stronger when we take into account dataset $\mathcal{S}_{2}$, however for the set $\mathcal{S}_{3}$ the constraints are similar to these obtained for the dataset $\mathcal{S}_{1}$.

\section{Discussion}

To discuss the limit on $\Delta_{\mathrm{g}}$ obtained above we will consider two cases: (i) emission from a polar cap, and (ii) emission from the accretion column. In the first case the upper limit on the $\Delta_{\mathrm{g}}$ obtained above can be interpreted as a limit on the compactness of the star. We plot the amount of gravitational light deflection as a function of the emission angle for a few values of the ratio of the neutron star radius to the gravitational radius $r / r_{\mathrm{g}}$ in Fig. 8. The maximal gravitational light deflection of about $30^{\circ}$ corresponds to the case with ratio $r / r_{\mathrm{g}} \approx 3$ for the photons emitted parallel to the surface. The limit on $\Delta_{\mathrm{g}}$ can be understood as the upper limit on the compactness of the neutron stars. In Fig. 9 we plot the ratio $r / r_{\mathrm{g}}$ as a function of the stellar gravitational mass for a few representative equations of state. The ratio $r / r_{\mathrm{g}}$ decreases with increasing mass. The upper limit on the ratio $r / r_{\mathrm{g}}$ corresponds for a given equation of state to the lower limit on the mass of the accreting neutron stars. Masses of neutron stars in some accreting objects have been estimated from the observations of binary motion, e.g. in the case of 4U1538-52 (Pakull et al. 1983; Reynolds et al. 1992) and they all seem to be consistent with the fiducial $1.4 M_{\odot}$ with some uncertainty. The general consensus seems to be that the

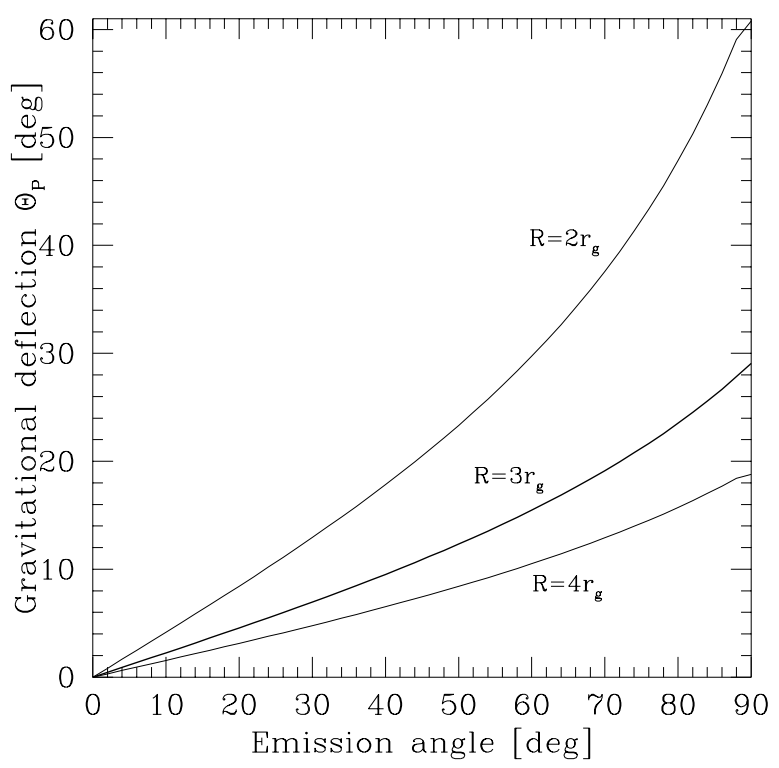

Fig. 8. The value of the gravitational light bending as a function of the emission angle, measured from the radial direction for photons emitted at different initial radii. The maximum gravitational light deflection is the value of deflection for the initial angle of $90^{\circ}$, i.e. parallel to the surface.

masses of all neutron stars are consistent with the mean value of $\approx 1.4 M_{\odot}$, and this is also the value found for the binary pulsars (Thorsett \& Chakrabarty 1999), however in LMXBs this range may be much wider and reach above $2 M_{\odot}$ (Bulik et al. 2000). If the neutron stars have masses around $1.4 M_{\odot}$ and the beams are wide then our results point to the very stiff equations of state e.g. SDB (Sahu et al. 1993).

A second possibility is that we see the emission from the accretion shock above the surface of the neutron star with the typical properties of the fan beam and that this emission is responsible for the observed flux at angles above $90^{\circ}$ with respect to the normal. Our considerations above imply that the emission is visible up to the angles exceeding the perpendicular to the normal by $\Delta_{\mathrm{g}}<33^{\circ}$. In the non-relativistic case this means that such shocks can lie no higher than $\approx 0.2 r$ above the surface of the neutron star. If the effects of the gravitational 


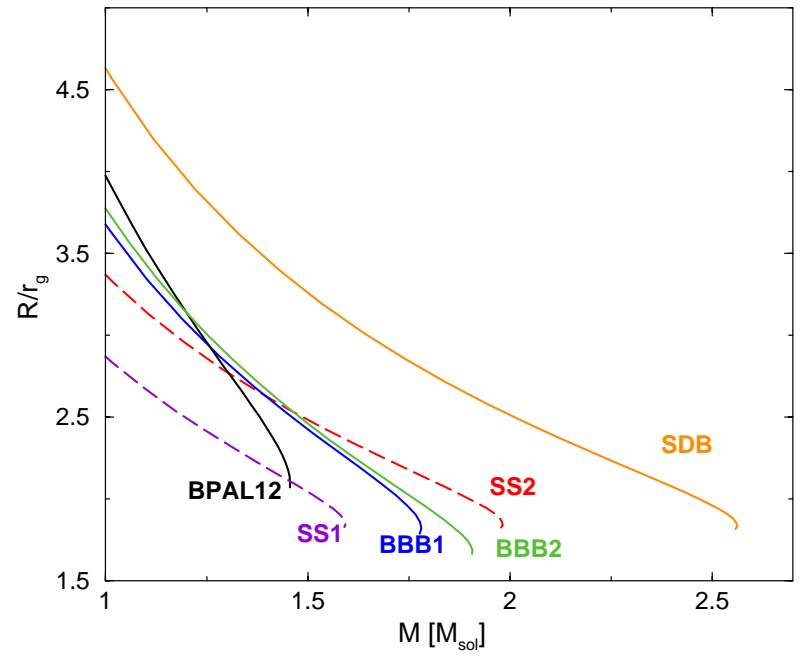

Fig. 9. The ratio of the stellar radius to the gravitational radius as a function of mass for several neutron star equations of state. The labels are BPAL12 - Prakash et al. (1997), SBD - Sahu et al. (1993), BB1 and BB2 - Baldo et al. (1997), while SS1 and SS2 correspond to the MIT Bag model of strange quark matter (Witten 1984) with two different densities at zero pressure.

light bending around a neutron star are included the maximum height allowed is even smaller.

We have obtained a limit on the possible inclination angles of the magnetic axis with respect to the rotation axis $\beta_{\max }<50^{\circ}$. A hint of such a distribution was already shown by Leahy (1991). He analyzed the lightcurves of 20 pulsars using a simple model with the beam dependence on the emission angle as $\cos ^{2} \phi$ and $\cos ^{4} \phi$ where $\phi$ is the direction to the normal of the surface. For each pulsar he found the angles $\theta$ and $\beta$, however the fits were degenerate in these angles, so that it was not clear a priori which angle is which. The resulting distribution was inconsistent with the assumption that both angles are random, and Leahy (1991) concluded that the mean angle between the magnetic and rotation axis is as small $15^{\circ}$. Inclusion of the gravitational light bending effects (Leahy \& Li 1995) does not alter this conclusion. Bulik et al. (1995) modeled lightcurves of $4 \mathrm{U} 1538-52$ and Vela X-1 observed by Ginga, and obtained the inclination angles below $50^{\circ}$ for their fits with best significance. Kraus et al. (1996) analyzed the pulse profiles of Cen X-3 and reconstructed the geometry and beam pattern for this object. They found that the most likely inclination angles of the magnetic axis to the rotation axis are $\sim 20^{\circ}$. An analysis of the pulse shape of Her X-1 (Blum \& Kraus 2000) also lead to a constraint on the angle between the magnetic and rotation axis $\beta<20^{\circ}$.

We have mentioned in Sect. 2 three special objects A0535-262, GX1+4, and RX J0812.4-3114 for which accretion flow eclipses have been reported (possibly this list also includes GS1843-24, Finger et al. 1999, and XTE J1946+274, Wilson et al. 2003). If such eclipses happen when the accretion column passes through the line of sight then they should take place when $|\theta-\beta|<\gamma$, where $\gamma$ is the angular width of the accretion column. The region corresponding to $\gamma=10^{\circ}$ is shaded in Fig. 1. We see that such eclipses should frequently take place in double pulsed light curves, if the magnetic axis is randomly oriented with respect to the rotation axis. Moreover within the single pulsed light curves a significant fraction, approximately $20-30 \%$ should exhibit such dips, and this fraction increases as the amount of gravitational light bending increases.

Yet the only three cases when such dips have been found are in single pulsed light curves. The lightcurve of A0535-262 seems to be double peaked, however Cemeljic \& Bulik (1998) pointed out that it can be interpreted as a single pulse divided by the accretion flow eclipse. This lack of double peaked pulsars with accretion flow eclipses (dips) may mean that the region of parameter space for double peaked light curves with dips is either empty or scarcely populated! Again we are drawn to the conclusion that the magnetic axis needs to be aligned with the rotation axis. If the magnetic axis inclination is restricted to the region $\beta_{\max }<50^{\circ}$, then one expects that the accretion flow eclipses to be observed only in the one pulsed lightcurves, as is the case. While this by itself is not a significant result, the identification of accretion flow eclipses is consistent with the conclusion that the range of allowed values of $\beta$ is limited.

One should mention here that there may possibly be a selection bias against seeing double pulsed objects. In the case of nearly perpendicular rotators the two pulses will be similar. It is therefore possible that the lack of double pulsed objects is due to misidentification of the pulse period which should be two times longer than reported. The fraction of such misidentified objects depends on the exact shape of the beam. For wide uncollimated beams the two pulses look similar for a range of angles $\beta$ as large as the width of the beams. However, our limit is $\beta_{\max }<50^{\circ}$, so the beams would have to be very wide. Additionally, the beam shape would have to be very fine tuned in order to compensate for the viewing geometry effects over such a large range of angles.

Another possible selection effect against seeing double peaked pulsars was shown by Beloborodov (2002). Assuming that both accretion caps lie exactly opposite one another on the surface of the neutron star and are identical,and that the radiation beam is well described by $F(\mu) \propto \mu$ (or intensity is independent of the angle of emission), he shows that the pulse shapes are flat in the region where both polar caps are visible due to gravitational light bending. This decreases the parameter space for seeing double peaked pulsars. However detailed modeling of individual objects tends to show that the above assumptions are not satisfied, the data usually requires non antipodal caps, complicated beam patterns, and sometimes differences between the two polar caps (Leahy \& Li 1995; Bulik et al. 1995; Kraus et al. 1996; Blum \& Kraus 2000).

The sample of accreting pulsars presented here is dominated by transient $\mathrm{Be} / \mathrm{X}$-ray systems. One should note that for the disk-fed persistent sources (Her X-1, SMC X-1, SMC X-2, Cen X-3, LMC X-4, RX J0648.1-4419) there are three double peaked objects out of six sources, still below the expected $\approx 79$ percent, but this is not a significant discrepancy. In the case of wind-fed persistent sources (Vela X-1, 1E 1145.1-6141, $4 \mathrm{U} 1907+09$, 4U1583-53, GX 301-2, 2S 0114+650) there are four double peaked out of six objects. However, because of the small number of these sources it is impossible to obtain any significant conclusion just from a statistical analysis and, as we 
already mentioned above, detailed modeling of two wind-fed pulsars (4U 1538-52 and Vela X-1) and one disk-fed (Cen X-3) resulted in low values of $\beta$.

\section{Conclusions}

We analyzed the shapes of accretion powered pulsar light curves, looking for the expected fraction of single and double peaked light curves, as well as these exhibiting accretion flow eclipses. We find that the expected number of single peaked lightcurves is small; certainly less than about one in five lightcurves should be single peaked both in the framework of the pencil beam model and a simple fan beam model. A general fan beam model predicts predominantly triple and quadruple peaked pulse shapes and only a small percentage of single peaked lightcurves. On the other hand the observations show that quite a large fraction of accreting pulsars have single peaked lightcurves in hard X-rays with the rest being double peaked. In order to explain this discrepancy between theory and observations we are drawn to conclude that the magnetic axes in accretion-powered pulsars tend to be aligned with the rotation axis. Such alignment decreases the expected number of double peaked lightcurves, as seen in Fig. 1. We have compared the models parametrized by the maximal inclination of the magnetic to the rotation axis $\beta_{\max }$ and the value of the maximum gravitational light deflection $\Delta_{\mathrm{g}}$ with the data. We obtain upper limits at the $99 \%$ confidence level $\beta_{\max }<50^{\circ}$ and $\Delta_{\mathrm{g}}<33^{\circ}$.

Our results are based on the classification of the neutron star lightcurves as single or double pulsed. In order to avoid possible misclassification we have attempted to use the data above $10 \mathrm{keV}$, or above the cyclotron line wherever possible. Moreover, we have attempted to classify as many pulsars as double when we were in doubt. In order to rid ourselves of the possible systematic effects we have repeated the analysis for the three datasets, and in the third dataset we included all the unclassifiable pulsars as double peaked objects to be conservative. The deficit of double pulsed objects is significant for every dataset that we analyzed. Moreover we note that the paucity of double peaked pulsars is so strong that our results still hold even if there are still a few misidentifications in our analysis. We have also shown that if the pulsar beam is strongly collimated, the deficit of double peaked objects becomes more significant.

Acknowledgements. We thank Peter Jonker, Biswajit Paul, and Silvia Zane for helpful comments on the contents of the Tables, and the anonymous referee for helpful comments on this paper. This research has been supported by the KBN grants 5P03D01721 and PBZ-KBN-054/P03/2001, and the EU Programme "Improving the Human Research Potential and the Socio-Economic Knowledge Base" (Research Training Network Contract HPRN-CT-2000-00137). TB is grateful for the hospitality of the Observatoire de Paris in Meudon and for the support of the CNRS/PAN Jumelage programme.

\section{References}

Angelini, L., Church, M. J., Parmar, A. N., Balucinska-Church, M., \& Mineo, T. 1998, A\&A, 339, L41
Aoki, T., Dotani, T., Ebisawa, K., et al. 1992, PASJ, 44, 641

Baldo, M., Bombaci, I., \& Burgio, G. F. 1997, A\&A, 328, 274

Beloborodov, A. M. 2002, ApJ, 566, L85

Bildsten, L., Chakrabarty, D., Chiu, J., et al. 1997, ApJS, 113, 367

Blum, S., \& Kraus, U. 2000, ApJ, 529, 968

Bulik, T., Kluzniak, W., \& Zhang, W. 2000, A\&A, 361, 153

Bulik, T., Meszaros, P., Woo, J. W., Hagase, F., \& Makishima, K. 1992, ApJ, 395, 564

Bulik, T., Riffert, H., Meszaros, P., et al. 1995, ApJ, 444, 405

Burderi, L., Di Salvo, T., Robba, N. R., La Barbera, A., \& Guainazzi, M. 2000, ApJ, 530, 429

Caraveo, P. A., Bignami, G. F., \& Goldwurm, A. 1989, ApJ, 338, 338

Cemeljic, M., \& Bulik, T. 1998, Acta Astronomica, 48, 65

Chakrabarty, D., Bildsten, L., Grunsfeld, J. M., et al. 1997, ApJ, 474, 414

Chakrabarty, D., Koh, T., Bildsten, L., et al. 1995, ApJ, 446, 826

Coe, M. J., Roche, P., Everall, C., et al. 1994, A\&A, 289, 784

Corbet, R., Marshall, F. E., Lochner, J. C., Ozaki, M., \& Ueda, Y. 1998, IAU Circ., 6803

Corbet, R. H. D., Finley, J. P., \& Peele, A. G. 1999a, ApJ, 511, 876

Corbet, R. H. D., Marshall, F. E., Coe, M. J., Laycock, S., \& Handler, G. 2001, ApJ, 548, L41

Corbet, R. H. D., Marshall, F. E., Peele, A. G., \& Takeshima, T. 1999b, ApJ, 517, 956

Corbet, R. H. D., \& Peele, A. G. 1997, ApJ, 489, L83

Cropper, M., Zane, S., Ramsay, G., Haberl, F., \& Motch, C. 2001, A\&A, 365, L302

Cusumano, G., Israel, G. L., Mannucci, F., et al. 1998, A\&A, 337, 772

Cusumano, G., Maccarone, M. C., Nicastro, L., Sacco, B., \& Kaaret, P. 2000, ApJ, 528, L25

Dennerl, K., Haberl, F., \& Pietsch, W. 1995, IAU Circ., 6184

Finger, M. H., Bildsten, L., Chakrabarty, D., et al. 1999, ApJ, 517, 449

Finger, M. H., Koh, D. T., Nelson, R. W., et al. 1996, Nature, 381, 291

Finger, M. H., Macomb, D. J., Lamb, R. C., et al. 2001, ApJ, 560, 378

Giacconi, R., Gursky, H., Kellogg, E., Schreier, E., \& Tananbaum, H. 1971, ApJ, 167, L67

Giacconi, R., Murray, S., Gursky, H., et al. 1972, ApJ, 178, 281

Giles, A. B., Galloway, D. K., Greenhill, J. G., Storey, M. C., \& Wilson, C. A. 2000, ApJ, 529, 447

Grevenev, S. A., Pavlinsk, M. N., \& Syunyaev, R. A. 1992, SvA Lett., 18,228

Haberl, F., Dennerl, K., Pietsch, W., \& Reinsch, K. 1997, A\&A, 318, 490

Hall, T. A., Finley, J. P., Corbet, R. H. D., \& Thomas, R. C. 2000, ApJ, 536,450

Huckle, H. E., Mason, K. O., White, N. E., et al. 1977, MNRAS, 180, 21P

Hughes, J. P. 1994, ApJ, 427, L25

Hulleman, F., in 't Zand, J. J. M., \& Heise, J. 1998, A\&A, 337, L25

Imanishi, K., Yokogawa, J., Tsujimoto, M., \& Koyama, K. 1999, PASJ, 51, L15

in 't Zand, J. J. M., Baykal, A., \& Strohmayer, T. E. 1998, ApJ, 496, 386

Isenberg, M., Lamb, D. Q., \& Wang, J. C. L. 1998, ApJ, 505, 688

Israel, G. L., Angelini, L., Campana, S., et al. 1998, MNRAS, 298, 502

Israel, G. L., Campana, S., Covino, S., et al. 2000, ApJ, 531, L131

Israel, G. L., Mereghetti, S., \& Stella, L. 1994, ApJ, 433, L25

Israel, G. L., Stella, L., Angelini, L., White, N. E., \& Giommi, P. 1995, IAU Circ., 6277

Israel, G. L., Stella, L., Angelini, L., et al. 1997, ApJ, 474, L53

Iwasawa, K., Koyama, K., \& Halpern, J. P. 1992, PASJ, 44, 9 
Jonker, P. G., \& van der Klis, M. 2001, ApJ, 553, L43

Kelley, R. L., Rappaport, S., \& Ayasli, S. 1983, ApJ, 274, 765

Kinugasa, K., Torii, K., Hashimoto, Y., et al. 1998, ApJ, 495, 435

Koh, D. T., Bildsten, L., Chakrabarty, D., et al. 1997, ApJ, 479, 933

Koyama, K., Kawada, M., Kunieda, H., Tawara, Y., \& Takeuchi, Y. 1990a, Nature, 343, 148

Koyama, K., Kawada, M., Takeuchi, Y., et al. 1990b, ApJ, 356, L47

Koyama, K., Kunieda, H., Takeuchi, Y., \& Tawara, Y. 1990c, PASJ, 42, L59

Koyama, K., Kawada, M., Tawara, Y., et al. 1991a, ApJ, 366, L19

Koyama, K., Kunieda, H., Takeuchi, Y., \& Tawara, Y. 1991b, ApJ, 370, L77

Kraus, U., Blum, S., Schulte, J., Ruder, H., \& Meszaros, P. 1996, ApJ, 467, 794

Lamb, D. Q., Wang, J. C. L., \& Wasserman, I. M. 1990, ApJ, 363, 670

Leahy, D. A. 1991, MNRAS, 251, 203

Leahy, D. A., \& Li, L. 1995, MNRAS, 277, 1177

Levine, A., Rappaport, S., Deeter, J. E., Boynton, P. E., \& Nagase, F. 1993, ApJ, 410, 328

Liu, Q. Z., van Paradijs, J., \& van den Heuvel, E. P. J. 2000, A\&AS, 147,25

Liu, Q. Z., van Paradijs, J., \& van den Heuvel, E. P. J. 2001, A\&A, 368,1021

Lochner, J. C., Marshall, F. E., Whitlock, L. A., \& Brandt, N. 1998, IAU Circ., 6814

Macomb, D. J., Finger, M. H., Harmon, B. A., Lamb, R. C., \& Prince, T. A. 1999, ApJ, 518, L99

Makishima, K., Ohashi, T., Kawai, N., et al. 1990, PASJ, 42, 295

Marsden, D., Gruber, D. E., Heindl, W. A., Pelling, M. R., \& Rothschild, R. E. 1998, ApJ, 502, L129

McClintock, J. E., Nugent, J. J., Li, F. K., \& Rappaport, S. A. 1977, ApJ, 216, L15

Mereghetti, S., Tiengo, A., Israel, G. L., \& Stella, L. 2000, A\&A, 354, 567

Meszaros, P. 1992, High-energy radiation from magnetized neutron stars Meszaros (Chicago: University of Chicago Press)

Meszaros, P., \& Nagel, W. 1985, ApJ, 298, 147

Nagase, F. 1989, PASJ, 41, 1

Oosterbroek, T., Orlandini, M., Parmar, A. N., et al. 1999, A\&A, 351, L33

Pakull, M., van Amerongen, S., Bakker, R., \& van Paradijs, J. 1983, A\&A, 122, 79

Paul, B., Agrawal, P. C., Mukerjee, K., et al. 2001, A\&A, 370, 529

Paul, B., \& Rao, A. R. 1998, A\&A, 337, 815

Prakash, M., Bombaci, I., Prakash, M., et al. 1997, Phys. Rep., 280, 1

Reig, P., \& Coe, M. J. 1999, MNRAS, 302, 700

Reig, P., \& Roche, P. 1999a, MNRAS, 306, 100

Reig, P., \& Roche, P. 1999b, MNRAS, 306, 95

Remillard, R., Levine, A., Takeshima, T., et al. 1998, IAU Circ., 6826

Reynolds, A. P., Bell, S. A., \& Hilditch, R. W. 1992, MNRAS, 256, 631
Robba, N. R., Burderi, L., Wynn, G. A., Warwick, R. S., \& Murakami, T. 1996, ApJ, 472, 341

Robba, N. R., \& Warwick, R. S. 1989, ApJ, 346, 469

Sahu, P. K., Basu, R., \& Datta, B. 1993, ApJ, 416, 267

Santangelo, A., Cusumano, G., dal Fiume, D., et al. 1998, A\&A, 338, L59

Santangelo, A., Segreto, A., Giarrusso, S., et al. 1999, ApJ, 523, L85

Saraswat, P., \& Apparao, K. M. V. 1992, ApJ, 401, 678

Saraswat, P., Mihara, T., Corbet, R., \& Ebisawa, K. 1997, in All-Sky X-Ray Observations in the Next Decade, 109

Schmidtke, P. C., Cowley, A. P., McGrath, T. K., \& Anderson, A. L. 1995, PASP, 107, 450

Schwentker, O. 1994, A\&A, 286, L47

Scott, D. M., Finger, M. H., Wilson, R. B., et al. 1997, ApJ, 488, 831

Seward, F. D., Charles, P. A., \& Smale, A. P. 1986, ApJ, 305, 814

Shklovsky, I. S. 1967, ApJ, 148, L1

Skinner, G. K., Bedford, D. K., Elsner, R. F., et al. 1982, Nature, 297, 568

Stollberg, M. T., Finger, M. H., Wilson, R. B., et al. 1999, ApJ, 512, 313

Sugizaki, M., Nagase, F., Torii, K., et al. 1997, PASJ, 49, L25

Tananbaum, H., Gursky, H., Kellogg, E. M., et al. 1972, ApJ, 174, L143

Tawara, Y., Yamauchi, S., Awaki, H., et al. 1989, PASJ, 41, 473

Thorsett, S. E., \& Chakrabarty, D. 1999, ApJ, 512, 288

Torii, K., Kinugasa, K., Katayama, K., et al. 1998a, ApJ, 508, 854

Torii, K., Kinugasa, K., Katayama, K., Tsunemi, H., \& Yamauchi, S. 1998b, ApJ, 503, 843

Torii, K., Sugizaki, M., Kohmura, T., Endo, T., \& Nagase, F. 1999, ApJ, 523, L65

Tsujimoto, M., Imanishi, K., Yokogawa, J., \& Koyama, K. 1999, PASJ, 51, L21

Ueno, M., Yokogawa, J., Imanishi, K., \& Koyama, K. 2000, PASJ, 52, L63

Wijnands, R., \& van der Klis, M. 1998, Nature, 394, 344

Wilson, C. A., \& Finger, M. H. 1998, IAU Circ., 7048

Wilson, C. A., Finger, M. H., Coe, M., \& Negueruela, I. 2003, ApJ, 584,996

Wilson, C. A., Finger, M. H., Harmon, B. A., et al. 1997, ApJ, 479, 388

Witten, E. 1984, Phys. Rev., 30, 272

Woo, J. W., Clark, G. W., Levine, A. M., Corbet, R. H. D., \& Nagase, F. 1996, ApJ, 467, 811

Yokogawa, J., Imanishi, K., Tsujimoto, M., Kohno, M., \& Koyama, K. 1999, PASJ, 51, 547

Yokogawa, J., \& Koyama, K. 1998, IAU Circ., 7028

Yokogawa, J., Imanishi, K., Ueno, M., \& Koyama, K. 2000a, PASJ, 52, L73

Yokogawa, J., Paul, B., Ozaki, M., et al. 2000b, ApJ, 539, 191 